Mosenzon, O. et al. (2019) Efficacy and safety of oral semaglutide in patients with type 2 diabetes and moderate renal impairment (PIONEER 5): a placebo-controlled, randomised, phase 3a trial. Lancet Diabetes and Endocrinology, 7(7), pp. 515-527. (doi: 10.1016/S2213-8587(19)30192-5)

There may be differences between this version and the published version. You are advised to consult the publisher's version if you wish to cite from it.

http://eprints.gla.ac.uk/210331/

Deposited on: 17 February 2020

Enlighten - Research publications by members of the University of Glasgow http://eprints.gla.ac.uk 


\section{Efficacy and safety of oral semaglutide in patients with type 2 diabetes and moderate renal impairment (PIONEER 5): a placebo-controlled, randomised, phase $3 a$ trial}

Ofri Mosenzon, Thalia Marie Blicher, Signe Rosenlund, Jan W Eriksson, Simon Heller, Ole Holm Hels, Richard Pratley, Thozhukat Sathyapalan, Cyrus Desouza, for the PIONEER 5 Investigators

Lancet Diabetes Endocrinol 2019

Published Online: June 9, 2019 http://dx.doi.org/10.1016/S2213-8587(19)30192-5.

See Online/Comment: http://dx.doi.org/10.1016/S2213-8587(19)30182-2.

Diabetes Unit, Division of Internal Medicine, Hadassah Hebrew University Hospital, Jerusalem, Israel (O Mosenzon MD); Novo Nordisk A/S, Søborg, Denmark (T M Blicher MD, S Rosenlund MD, O H Hels PhD); Clinical Diabetology and Metabolism, Department of Medical Sciences, Uppsala University, Uppsala, Sweden (Prof J W Eriksson MD); Academic Unit of Diabetes, Endocrinology and Metabolism, Department of Oncology and Metabolism, University of Sheffield, Sheffield, UK (Prof S Heller MD); AdventHealth Translational Research Institute for Metabolism and Diabetes, Orlando, FL, USA (Prof R Pratley MD); Academic Diabetes, Endocrinology and Metabolism Research Group, Hull York Medical School, University of Hull, Hull, UK (Prof T Sathyapalan MD); and Division of Diabetes, Endocrinology, and Metabolism, Department of Internal Medicine, University of Nebraska Medical Center, Omaha, NE, USA (Prof C Desouza MBBS) Correspondence to: Dr Ofri Mosenzon, Diabetes Unit, Internal Medicine, Hadassah Hebrew University Hospital, Ein Kerem, PO Box 12000, Jerusalem 91120, Israel. ofrim@hadassah.org.il.

\section{Summary}

Background Oral semaglutide is the first oral glucagon-like peptide-1 (GLP-1) receptor agonist for glycaemic control in patients with type 2 diabetes. Type 2 diabetes is commonly associated with renal impairment, restricting treatment options. We aimed to investigate the efficacy and safety of oral semaglutide in patients with type 2 diabetes and moderate renal impairment.

Methods This randomised, double-blind, phase $3 a$ trial was undertaken at 88 sites in eight countries. Patients aged 18 years and older, with type 2 diabetes, an estimated glomerular filtration rate of 30-59 mL/min per $1.73 \mathrm{~m}^{2}$, and who had been receiving a stable dose of metformin or sulfonylurea, or both, or basal insulin with or without metformin for the past 90 days were eligible. Participants were randomly assigned (1:1) by use of an interactive web-response system, with stratification by glucose-lowering medication and renal function, to receive oral semaglutide (dose escalated to $14 \mathrm{mg}$ once daily) or matching placebo for 26 weeks, in addition to background medication. Participants and site staff were masked to assignment. Two efficacy-related estimands were defined: treatment policy (regardless of treatment discontinuation or rescue medication) and trial product (on treatment without rescue medication) in all participants randomly assigned. Endpoints were change from baseline to week 26 in $\mathrm{HbA}_{1 c}$ (primary endpoint) and bodyweight (confirmatory secondary endpoint), assessed in all participants with sufficient data. Safety was assessed in all participants who received at least one dose of study drug. This trial is registered on ClinicalTrials.gov, number NCT02827708, and the European Clinical Trials Registry, number EudraCT 2015-005326-19, and is now complete. 
Findings Between Sept 20, 2016, and Sept 29, 2017, of 721 patients screened, 324 were eligible and randomly assigned to oral semaglutide $(n=163)$ or placebo $(n=161)$. Mean age at baseline was 70 years (SD 8), and 168 $(52 \%)$ of participants were female. 133 (82\%) participants in the oral semaglutide group and 141 (88\%) in the placebo group completed 26 weeks on treatment. At 26 weeks, oral semaglutide was superior to placebo in decreasing $\mathrm{HbA}_{1 \mathrm{c}}$ (estimated mean change of -1.0 percentage point (SE $0.1 ;-11 \mathrm{mmol} / \mathrm{mol}$ [SE 0.8]) vs -0.2 percentage points (SE $0.1 ;-2 \mathrm{mmol} / \mathrm{mol}$ [SE 0.8]); estimated treatment difference [ETD]: -0.8 percentage points, $95 \% \mathrm{Cl}-1 \cdot 0$ to -0.6 ; $\mathrm{p}<0.0001$ ) and bodyweight (estimated mean change of $-3.4 \mathrm{~kg}$ [SE 0.3] vs -0.9 kg [SE 0.3]; ETD, $-2.5,95 \% \mathrm{Cl}-3.2$ to $-1 \cdot 8 ; p<0.0001$ ) by the treatment policy estimand. Significant differences were seen for the trial product estimand: mean change in $\mathrm{HbA}_{1 \mathrm{c}}-1.1$ percentage points (SE 0.1; $-12 \mathrm{mmol} / \mathrm{mol}$ [SE 0.8] versus -0.1 percentage points (SE $0.1 ;-1 \mathrm{mmol} / \mathrm{mol}$ [SE 0.8]; ETD -1.0 percentage points, $95 \% \mathrm{Cl}-1.2$ to $-0.8 ; \mathrm{p}<0.0001$ ); mean change in bodyweight $-3.7 \mathrm{~kg}$ (SE 0.3) versus $-1.1 \mathrm{~kg}$ (SE 0.3; ETD -2.7 kg, 95\% Cl -3.5 to -1.9; p<0.0001). More patients taking oral semaglutide than placebo had adverse events (120 [74\%] of 163 vs 105 [65\%] of 161), and discontinued treatment as a result (24 [15\%] vs eight [5\%]). Gastrointestinal events, mainly mild-to-moderate nausea, were more common with oral semaglutide than with placebo. Three deaths occurred during the treatment period that were not considered to be treatment related, one in the semaglutide group and two in the placebo group.

Interpretation Oral semaglutide was effective in patients with type 2 diabetes and moderate renal impairment, potentially providing a new treatment option for this population. Safety, including renal safety, was consistent with the GLP-1 receptor agonist class.

Funding Novo Nordisk A/S.

Copyright (c) 2019 Elsevier Ltd. All rights reserved. 


\section{Research in context}

\section{Evidence before this study}

Some glucagon-like peptide-1 (GLP-1) receptor agonists have shown effective glycaemic control in patients with type 2 diabetes and impaired renal function (eg, liraglutide was superior to placebo, and dulaglutide was similarly effective to insulin, without a negative effect on renal function in either case). However, GLP-1 receptor agonists are all administered subcutaneously, which might not be ideal for some patients. We searched PubMed articles on Jan 17, 2019, published in the past 5 years, with no language restrictions, using the search terms "type 2 diabetes", "renal impairment", and "chronic kidney disease". We reviewed the prescribing information documents of approved glucoselowering drugs for dose adjustments, contraindications, or both, associated with renal impairment. Type 2 diabetes is often associated with renal impairment, but common oral glucose-lowering drugs have restrictions for use (eg, sodiumglucose co-transporter-2 inhibitors), require increased monitoring (eg, metformin) or dose adjustment (eg, most dipeptidyl peptidase-4 inhibitors), or are associated with an increased risk of hypoglycaemia and weight gain (sulfonylureas) in patients with decreased kidney function. Chronic kidney disease is a risk factor for hypoglycaemia, but many patients with renal impairment might be using insulin, sulfonylureas, or glinides, or a combination of these for glycaemic control, which are also associated with an increased risk of hypoglycaemia and weight gain. Therefore, a need exists for improved glucose-lowering treatment options for these patients.

\section{Added value of this study}

Oral semaglutide escalated to $14 \mathrm{mg}$ taken once daily was superior to placebo in decreasing $\mathrm{HbA}_{1 \mathrm{c}}$ and bodyweight in patients with type 2 diabetes and moderate renal impairment who were uncontrolled on metformin, sulfonylureas, or both, or basal insulin with or without metformin. The proportion of patients who achieved a target of $\mathrm{HbA}_{1 \mathrm{c}}$ of less than $7.0 \%(53 \mathrm{mmol} / \mathrm{mol}$ ) was higher with oral semaglutide than with placebo. Considering the population, oral semaglutide showed a similar overall safety profile to that seen previously with the GLP-1 receptor agonist class and did not adversely affect renal function.

\section{Implications of all the available evidence}

Oral semaglutide is the first orally available GLP-1 receptor agonist and has the potential to expand the treatment options for patients with type 2 diabetes and moderate renal impairment, for whom current oral glucose-lowering treatment options are restricted. Further and larger studies are needed to better define the place of oral semaglutide, including any potential renoprotective effect, in the treatment of patients with type 2 diabetes and moderate renal failure. 


\section{Introduction}

Glucagon-like peptide-1 (GLP-1) receptor agonists act on multiple pathophysiological defects present in people with type 2 diabetes. ${ }^{1}$ Semaglutide is a GLP-1 analogue currently approved for once-weekly subcutaneous injection for treatment of type 2 diabetes, ${ }^{2}$ and has been shown to decrease $\mathrm{HbA}_{1 \mathrm{c}}$ and bodyweight effectively in patients who have uncontrolled hyperglycaemia on one or more oral glucose-lowering drugs. ${ }^{3}$ Elimination of semaglutide occurs via multiple pathways involving both the liver and kidneys. ${ }^{4}$

An oral semaglutide tablet has been developed, in which semaglutide is coformulated with the absorption enhancer, sodium N-(8-[2-hydroxybenzoyl]amino) caprylate, to facilitate semaglutide absorption across the gastric mucosa. ${ }^{5}$ Oral semaglutide monotherapy has shown significant decreases in $\mathrm{HbA}_{1 \mathrm{c}}$ and bodyweight compared with placebo in patients with type 2 diabetes not controlled through diet and exercise,${ }^{6}$ and compared with sitagliptin in addition to background metformin with or without sulfonylurea. ${ }^{7}$

Type 2 diabetes is commonly associated with renal impairment, which restricts the use of some glucose lowering medications and makes achieving treatment targets challenging. Insulin and sulfonylureas are associated with weight gain and increased risk of hypoglycaemia in all patients, and even more so in those with chronic kidney disease..$^{8,9}$ Metformin can be used with caution in patients with an estimated glomerular filtration rate (eGFR) of at least $30 \mathrm{~mL} / \mathrm{min}$ per $1.73 \mathrm{~m}^{2}$ despite its association with lactic acidosis-a rare but serious complication. ${ }^{10}$ Most sodiumglucose co-transporter- 2 (SGLT2) inhibitors are not recommended in patients with creatinine clearance of less than $45 \mathrm{~mL} / \mathrm{min} .{ }^{11}$ Dipeptidyl peptidase-4 (DPP-4) inhibitors can be used in patients with chronic kidney disease (all stages, except stage 5 for saxagliptin), although these drugs (with the exception of linagliptin) must be given at a decreased dose dependent on the stage of chronic kidney disease. ${ }^{11}$ By contrast, the GLP-1 receptor agonists semaglutide, liraglutide, albiglutide, and dulaglutide do not require dose adjustment in patients with chronic kidney disease at stage 4 and above, and could be useful alternatives to other glucose-lowering drugs. ${ }^{11}$ Nevertheless, this class of medication is only available for subcutaneous injection, which might not be ideal for some patients.

In people without diabetes, the pharmacokinetics of oral semaglutide were not affected by renal impairment. ${ }^{12}$ However, the efficacy and safety profile of oral semaglutide in patients with diabetes and renal impairment is unknown. In this phase 3a trial (PIONEER 5), we compared the efficacy and safety of once-daily oral semaglutide with placebo, in addition to existing background medication, in patients with type 2 diabetes and moderate renal impairment (eGFR of 30-59 $\mathrm{mL} / \mathrm{min}$ per $1.73 \mathrm{~m}^{2}$ ).

\section{Methods}

\section{Study design}

This multicentre, double-blind, randomised, placebo controlled, phase $3 a$ trial was carried out at 88 sites in Denmark, Finland, Israel, Poland, Russia, Sweden, the UK, and the USA. Two different scientific questions associated with efficacy objectives were addressed through the definition of two estimands: treatment policy and trial product. Both estimands were defined on the basis of interactions with regulatory agencies. The treatment policy estimand was the protocol-defined primary estimand. Superiority testing was based on only the treatment policy estimand, and results were controlled for multiplicity. The trial product estimand was the protocol-defined secondary estimand and was used to assess the magnitude of an established treatment effect.

The treatment policy estimand assesses the treatment effect for all randomly assigned participants, regardless of trial product discontinuation or use of rescue medication. This estimand reflects the intention-to-treat principle as 
defined in International Conference on Harmonisation Guidelines for Good Clinical Practice (ICH) E9. ${ }^{13}$ The estimand reflects the effect of initiating treatment with oral semaglutide compared with initiating treatment with placebo, both potentially followed by either discontinuation of trial product or addition of or switch to another glucose-lowering drug.

The trial product estimand assesses the treatment effect for all randomly assigned participants, under the assumption that all participants remained on study drug (oral semaglutide or placebo) for the entire planned duration of the trial and did not use rescue medication. This estimand aims to reflect the effect of oral semaglutide compared with placebo without the confounding effect of rescue medication. The statistical analysis that was applied to this estimand is similar to that applied to many phase $3 a$ diabetes trials. Results from such analyses are included in many product labels (eg, European summary of product characteristics [SmPC]) for glucose-lowering drugs (eg, the SmPC for Ozempic $\left.{ }^{14}\right)$.

Discontinuation of assigned study drug and initiation of rescue medication are accounted for by the treatment policy strategy for the treatment policy estimand, and by the hypothetical strategy for the trial product estimand as defined in draft ICH E9(R1). ${ }^{15}$ Further details on the estimands are in the appendix (pp 5-6).

Approval was secured from the relevant Institutional Review board or Independent Ethics Committee for each site before trial commencement, which was done according to applicable national requirements and in compliance with ICH E6 and the Declaration of Helsinki. A redacted version of the protocol is in the appendix (pp 29-195).

\section{Participants}

Eligible patients were aged 18 years or older, with type 2 diabetes (diagnosed $\geq 90$ days before screening), had a $\mathrm{HbA}_{1 \mathrm{c}}$ of $7 \cdot 0-9.5 \%$ (53-80 mmol/mol) and moderate renal impairment (Chronic Kidney Disease-Epidemiology Collaboration [CKD-EPI] stage 3), ${ }^{16}$ defined as an eGFR of 30-59 mL/min per $1.73 \mathrm{~m}^{2}$, calculated by use of the CKDEPI formula). ${ }^{17}$ Patients were required to be able to provide informed consent and be treated with stable doses of one of the following regimens for 90 days before screening: metformin ( $\geq 1500 \mathrm{mg}$ or maximum tolerated dose), a sulfonylurea (at least half of the maximum approved dose or maximum tolerated dose), or both; or basal insulin with or without metformin. Key exclusion criteria included: rapidly progressing renal disease (as judged by the investigator) or known nephrotic albuminuria (>2200 mg per $24 \mathrm{~h}$ or $>2200 \mathrm{mg} / \mathrm{g}$ ); family or personal history of multiple endocrine neoplasia type 2 or medullary thyroid carcinoma; history of malignant neoplasms within the past 5 years; history of pancreatitis; myocardial infarction, stroke, or admission to hospital for unstable angina or transient ischaemic attack within the past 180 days, or New York Heart Association Class IV heart failure; ${ }^{18}$ or proliferative retinopathy or maculopathy (determined by fundus photography or dilated fundoscopy 90 days or fewer before randomisation and requiring acute treatment). Full eligibility criteria are detailed in the appendix (pp 2-3).

All participants provided written informed consent before conduct of any trial-related activity.

\section{Randomisation and masking}

Participants were randomly assigned (1:1) to receive either once-daily oral semaglutide (escalated to $14 \mathrm{mg}$ ) or placebo, in addition to their background medication (appendix p 16). Randomisation was done by use of an interactive web-response system, which allocated dispensing unit numbers for each patient. Randomisation was stratified by background glucose-lowering medication (metformin alone, sulfonylurea with or without metformin, or basal insulin with or without metformin) and renal function (eGFR $45-59 \mathrm{~mL} / \mathrm{min}$ per $1.73 \mathrm{~m}^{2}$ [CKD-EPI stage 3A] or $30-44 \mathrm{~mL} / \mathrm{min}$ per $1.73 \mathrm{~m}^{2}$ [stage $3 \mathrm{~B}$ ]; at least $40 \%$ of patients had to be at stage $3 \mathrm{~B}$ at screening). Patients and site 
staff were masked to assignment, which was maintained by use of visually identical oral semaglutide and placebo tablets.

\section{Procedures}

After a 2-week screening period, participants were randomly assigned to treatment with once-daily oral semaglutide or placebo for 26 weeks, with a follow-up period of 5 weeks (appendix $p$ 16). Oral semaglutide was initiated at a $3 \mathrm{mg}$ dose, then escalated to $7 \mathrm{mg}$ at 4 weeks, and $14 \mathrm{mg}$ at 8 weeks. No dose adjustment of study drug were permitted during the trial. The use of dose escalation intended to improve gastrointestinal tolerability, because previous studies have found gastrointestinal side-effects at high initial doses. ${ }^{19}$ Absorption of oral semaglutide is affected by food and fluid in the stomach, so participants in both groups were instructed to take the medication in the morning in a fasted state with up to half a glass of water (approximately $120 \mathrm{~mL}$ ), 30 min before any other food, beverage, or other oral medication.

Participants were required to continue background glucose-lowering medication throughout the trial. Those receiving metformin and sulfonylureas were required to maintain the same dose level and frequency as at trial entry; those receiving basal insulin were recommended to have the dose decreased by $20 \%$ after random assignment to treatment group to minimise the risk of hypoglycaemic episodes. Up-titration of basal insulin (to a dose not exceeding that at randomisation) was permitted in weeks 10-16, after the maximum dose of oral semaglutide was reached.

Patients with persistent and unacceptable hyperglycaemia were offered treatment intensification with rescue medication, prescribed at the investigator's discretion and as an add-on to assigned treatment, in accordance with international guidelines. ${ }^{20}$ Persistent or unacceptable hyperglycaemia was defined as a fasting plasma glucose concentration exceeding $13.3 \mathrm{mmol} / \mathrm{L}(240 \mathrm{mg} / \mathrm{dL})$ in weeks $12-16$ or $11.1 \mathrm{mmol} / \mathrm{L}(200 \mathrm{mg} / \mathrm{dL})$ from week 17 to end of treatment, assessed at a central laboratory (ICON Laboratory Services, Dublin, Ireland). Rescue medication was selected in accordance with American Diabetes Association (ADA) or European Association for the Study of Diabetes guidelines, excluding GLP-1 receptor agonists, DPP-4 inhibitors, or amylin analogues.

Blood samples were taken at baseline and weeks 4, 8, 14, 20, 26, and 31 for assessment of glucose metabolism, lipid profile, and other laboratory parameters. Bodyweight and BMI were also assessed at these visits. Renal function was assessed at baseline and weeks 14 and 26. Patient reported outcomes were recorded at baseline and weeks 8 and 26.

Patients who prematurely discontinued their allocated treatment (eg, due to adverse events) were switched to an appropriate locally approved treatment selected at the investigator's discretion, excluding GLP-1 receptor agonists. All participants were asked to complete the protocol-specified visit schedule, regardless of premature discontinuation of allocated treatment or use of rescue medication, unless consent was withdrawn.

We recorded adverse events at every visit, including a 5-week safety follow-up visit at week 31 or within 3 days of study drug discontinuation, and again 5 weeks after study drug discontinuation. All adverse events were coded using the Medical Dictionary for Regulatory Activities (version 20.1). Adverse events were defined as any unfavourable and unintended sign (including an abnormal laboratory finding), symptom, or disease temporally associated with the use of a study drug, whether or not considered related to the drug. A serious adverse event was defined as any event that resulted in death, a life-threatening event, inpatient treatment in hospital or extension of existing admission to hospital (full list of eligible events is in the appendix [p 4]). Details of other efficacy and safety assessments are provided in the appendix ( $p$ 4). An independent event adjudication committee (EAC) validated selected adverse events according to predefined diagnostic criteria, including cardiovascular events, in line with US Food and Drug Administration 
requirements. Masked adjudication was done for instances of acute coronary syndrome, acute kidney injury, acute pancreatitis, cerebrovascular events, death, heart failure requiring admission to hospital, lactic acidosis, malignant neoplasm, and malignant thyroid neoplasm or C-cell hyperplasia.

\section{Outcomes}

The primary endpoint was change from baseline to week 26 in $\mathrm{HbA}_{1 \mathrm{c}}$ and the confirmatory secondary endpoint was change from baseline to week 26 in bodyweight. Supportive secondary endpoints were achievement at week 26 of the targets of $\mathrm{HbA}_{1 \mathrm{c}}$ of less than $7.0 \%$ (53 mmol$\left./ \mathrm{mol}\right)^{21}$ and $6.5 \%(48 \mathrm{mmol} / \mathrm{mol})$ or under, ${ }^{22}$ and the following composite endpoints: $\mathrm{HbA}_{1 \mathrm{c}}$ less than $7 \cdot 0 \%$ without treatment-emergent symptomatic hypoglycaemia that was severe (American Diabetes Association [ADA] classification ${ }^{23}$ ) or confirmed by blood glucose concentration $(<3.1 \mathrm{mmol} / \mathrm{L}[56$ $\mathrm{mg} / \mathrm{dL}]$ ), without weight gain; and $\mathrm{HbA}_{1 \mathrm{c}}$ decrease of 1.0 percentage points or more with weight loss of $3 \%$ or more from baseline. Further secondary endpoints included weight loss of $5 \%$ or more and $10 \%$ or more; change from baseline to week 26 in fasting plasma glucose, BMI, waist circumference, fasting lipid profile, and C-reactive protein; and patient-reported outcomes on health-related quality of life (SF-36 version 2 Health Survey [acute version], and the status version of the Diabetes Treatment Satisfaction Questionnaire [DTSQs]).

Safety endpoints included the number of treatment emergent adverse events, and the number of symptomatic hypoglycaemic episodes that were severe or confirmed by blood glucose concentration ( $<3.1 \mathrm{mmol} / \mathrm{L}$ [56 mg/dL]), or both. Severe hypoglycaemia was defined as the participant requiring third-party assistance to administer corrective treatment (ADA classification). ${ }^{23}$ Additional safety endpoints included changes from baseline to week 26 in a range of laboratory assessments (including eGFR and urinary albumin to creatinine ratio [UACR]), electrocardiograms (ECGs), physical examinations, vital signs, and eye examinations.

\section{Statistical analysis}

We used descriptive statistics to summarise baseline demographic information for all participants who were randomly assigned to a study drug.

We calculated the sample size to ensure a power of $90 \%$ or more for testing superiority of oral semaglutide versus placebo in change of $\mathrm{HbA}_{1 \mathrm{c}}$ for the treatment policy estimand. Based on data from previous trials, we assumed a treatment effect of $-0.5 \%$ (SD 1.1) for $\mathrm{HbA}_{1 \mathrm{c}}$ for oral semaglutide versus placebo, requiring 324 patients to be randomly assigned (1:1) to treatment.

The endpoints were assessed through the treatment policy and trial product estimands.

We tested the superiority of oral semaglutide versus placebo in terms of change from baseline to week 26 in $\mathrm{HbA}_{1 \mathrm{c}}$ and bodyweight. We based the confirmation of efficacy of oral semaglutide using these measures, both from baseline to week 26, on a hierarchical testing strategy to control the overall type 1 error for the hypotheses assessed by use of the treatment policy estimand. We estimated the treatment policy estimand (analysis of covariance using data irrespective of discontinuation of trial product or initiation of rescue medication) using a pattern mixture model with multiple imputation to handle missing data at week 26 for both confirmatory endpoints. We imputed data in the groups defined by study drug and treatment status at week 26 . We based both the imputation and analysis on ANCOVA models, with region (Africa, Asia, Europe, North America, or South America), stratification factors, and the interaction between the two stratification factors as categorical fixed effects, and baseline $\mathrm{HbA}_{1 \mathrm{c}}$ measurement as a covariate. We combined the results using Rubin's rule. We estimated the trial product estimand using a mixed model 
for repeated measurements (MMRM) that used data collected before premature discontinuation of study drug or initiation of rescue medication from all participants who were allocated to treatment.

We analysed binary endpoints using a logistic regression model. For the treatment policy estimand, we imputed missing data similarly as for the continuous endpoints, whereas we imputed missing data for the trial product estimand from patients randomly allocated to the same study drug using a sequential multiple imputation method. Odds ratio [OR] calculations were evaluated for the treatment policy estimand and the trial product estimand using the full analysis set, and proportions of participants who achieved binary outcomes were calculated using the observed data (ie, those who attended the week 26 visit and contributed to the proportions). For the treatment policy estimand, we imputed missing data similarly as for the continuous endpoints, whereas we imputed missing data for the trial product estimand from participants randomly assigned to the same treatment group using a sequential multiple imputation method.

We assessed safety endpoints during the on-treatment and in-trial periods using data from all participants exposed to a study drug (the safety analysis set). The in-trial period was the period during which participants were considered to be in the trial, including the follow-up period, regardless of whether they had discontinued the study drug or used rescue medication; this period was used when reporting cardiovascular events, neoplasms, rare events, diabetic retinopathy, and deaths. The on-treatment period was the period during which the participant was treated with study drug plus an ascertainment window of 5 weeks (for adverse events and hypoglycaemia) or 3 days (for laboratory assessments, physical examinations, and vital signs) after the last date on study drug. We assessed laboratory assessments of physical examinations and vital signs until 3 days after the last date on study drug.

We did sensitivity analyses on the primary and confirmatory secondary endpoints, primarily to assess the effect of missing data. The evaluation of the robustness of the primary endpoint was mainly on the basis of a pattern mixturemodel approach using multiple imputation. We did post-hoc exploratory and descriptive investigations of the primary and secondary endpoints by eGFR strata (ie, chronic kidney disease stage 3A and stage 3B).

$p$ values are unadjusted two-sided $p$ values for the test of no difference. We did all analyses using SAS version 9.4M2. Further information on statistical methods are in the appendix ( $p$ 7). The trial is registered with the European Clinical Trials Database, EudraCT 2015-005326-19, and ClinicalTrials.gov, number NCT02827708.

Role of the funding source

The funder of the trial was involved in trial design, monitoring, data collection, data analysis, data interpretation, and writing of the report. The corresponding author had full access to all the data in the study and had final responsibility for the decision to submit for publication.

\section{Results}

Between Sept 20, 2016, and Sept 29, 2017, we screened 721 patients, of whom 324 were randomly assigned to oral semaglutide $(n=163)$ or placebo $(n=161$; figure 1). Baseline demographic and disease characteristics were similar between the two treatment groups (table 1; appendix p 8). Mean age was 70 years (SD 8 ) and 168 (52\%) of 324 participants were female. 128 (40\%) of participants had stage 3B chronic kidney disease and 242 (75\%) used metformin, 131 (40\%) used sulfonylureas, and 114 (35\%) used basal insulin at baseline (appendix p 9). 158 (97\%) of 163 participants in the oral semaglutide group and 156 (97\%) of 161 in the placebo group completed the trial, and $133(82 \%)$ in the oral semaglutide group and $141(88 \%)$ in the placebo group completed 26 weeks on treatment (figure 1). Of all participants, seven (4\%) in the oral semaglutide group and $16(10 \%)$ in the placebo group required 
rescue medication over the treatment period (appendix p 10); of those who completed treatment, most did so without the use of rescue medication (figure 1).

Oral semaglutide was superior to placebo in decreasing $\mathrm{HbA}_{1 c}$. Mean changes from baseline in $\mathrm{HbA}_{1 \mathrm{c}}$ at week 26 were $-1.0 \%$ (SE $0 \cdot 1 ;-11 \mathrm{mmol} / \mathrm{mol}$ [SE 0.8]) for oral semaglutide and $-0 \cdot 2 \%$ (SE 0.1; $-2 \mathrm{mmol} / \mathrm{mol}$ [SE 0.8]) for placebo when assessed using the treatment policy estimand (estimated treatment difference [ETD] $-0.8 \%, 95 \% \mathrm{Cl}-$ 1.0 to $-0.6 ; p<0.0001$; figure $2 A$ ). Similarly, when assessed using the trial product estimand, oral semaglutide provided a significantly greater decrease in $\mathrm{HbA}_{1 \mathrm{c}}$ than placebo did: mean change from baseline $-1 \cdot 1 \%$ (SE $0 \cdot 1 ;-12$ $\mathrm{mmol} / \mathrm{mol}$ [SE 0.8]) versus -0.1\% (SE 0.1; $-1 \mathrm{mmol} / \mathrm{mol}$ [SE 0.8]); ETD -1.0\%, 95\% Cl $-1 \cdot 2$ to $-0 \cdot 8 ; \mathrm{p}<0 \cdot 0001$; figure $2 \mathrm{~B}$ ).

Oral semaglutide was superior to placebo (ETD $-2.5 \mathrm{~kg}, 95 \% \mathrm{Cl}-3.2$ to $-1 \cdot 8 ; \mathrm{p}<0.0001$ ) in decreasing bodyweight. Mean change in bodyweight from baseline to week 26 was $-3.4 \mathrm{~kg}$ (SE 0.3$)$ for oral semaglutide and $-0.9 \mathrm{~kg}$ (SE 0.3) for placebo when assessed using the treatment policy estimand (figure 3A). A significant difference was also seen when assessed using the trial product estimand, with mean changes from baseline to week 26 of $-3 \cdot 7$ $\mathrm{kg}(\mathrm{SE} 0.3)$ for the oral semaglutide group and $-1.1 \mathrm{~kg}$ (SE 0.3) for the placebo group (ETD $-2.7 \mathrm{~kg}, 95 \% \mathrm{Cl}-3.5$ to $-1.9 ; p<0.0001$; figure 3B).

At 26 weeks, a greater proportion of participants achieved at least a 1.0\% decrease in $\mathrm{HbA}_{1 c}$ (treatment policy estimand: 93 [60\%] of 154 participants in semaglutide group vs 31 [20\%] of 155 in placebo group with non-missing data regardless of trial product discontinuation or rescue medication use; trial product estimand: 84 [67\%] of 126 in semaglutide group vs 24 [19\%] of 127 in placebo group with non-missing data who completed treatment without rescue medication; appendix p 19) and more achieved the targets of $\mathrm{HbA}_{1 \mathrm{c}}$ of less 7.0\% (89 [58\%] of 154 vs 35 [23\%] of 155) and 6.5\% or more (60 [39\%] vs 12 [8\%]) with oral semaglutide than with placebo (treatment policy estimand; appendix pp 20-21). The odds of achieving both targets for both estimands were significantly higher with oral semaglutide than with placebo $(p<0.0001)$.

By use of the treatment policy estimand, a higher proportion of patients achieved weight losses of $5 \%$ or more $(55$ [36\%] of 154 in semaglutide group vs 15 [10\%] of 155 in placebo group) and 10\% or more (13 [8\%] in semaglutide group vs none in placebo group) with oral semaglutide than with placebo; similar results were achieved with the trial product estimand (appendix p 11). Compared with placebo, the odds of achieving weight loss were significantly improved with oral semaglutide for thresholds of both $5 \%$ or more $(p<0.0001$ for both estimands) and $10 \%$ or more $(p=0.0086$ for treatment policy estimand; $p=0.0040$ for trial product estimand; appendix $p$ 11).

Compared with placebo, more participants achieved the composite endpoint of $\mathrm{HbA}_{1 \mathrm{c}}$ of less than $7 \cdot 0 \%$ without symptomatic hypoglycaemia that was severe or confirmed by blood glucose concentration without weight gain at week 26 (figure 4). Estimated ORs significantly favoured oral semaglutide compared with placebo when assessed by the treatment policy estimand (estimated OR $5 \cdot 74,95 \% \mathrm{Cl} 3 \cdot 25-10 \cdot 16 ; \mathrm{p}<0 \cdot 0001$ ) and the trial product estimand (9.04, 4.77-17.15; $\mathrm{p}<0.0001)$. The odds of achieving a decrease in $\mathrm{HbA}_{1 \mathrm{c}}$ of 1.0 percentage point or more with weight loss of $3 \%$ or more from baseline to week 26 were also significantly better with oral semaglutide than with placebo ( $p<0.0001$ for both estimands; appendix $p$ 22).

Data for the supportive secondary outcomes of fasting plasma glucose, BMI, waist circumference, C-reactive protein, and fasting lipids are in the appendix ( $\mathrm{p}$ 11). Outcomes for fasting plasma glucose, BMI, and waist circumference favoured oral semaglutide over placebo, whereas they were similar between treatments for C-reactive protein and fasting lipids.

Health-related quality of life, measured by use of SF-36 version 2 (acute version), significantly favoured oral semaglutide for the physical component summary (ETD 1.98, 95\% Cl 0.57-3.39; $\mathrm{p}=0.0058$ ) and the domains of role- 
physical $(2 \cdot 29,0.60-3 \cdot 98 ; p=0.0079)$, bodily pain $(2 \cdot 28,0 \cdot 19-4 \cdot 38 ; p=0 \cdot 0326)$, and social functioning $(1 \cdot 83,0 \cdot 13-$ 3.53; $p=0.035$ ) compared with placebo (treatment policy estimand; appendix p 23). Additionally, the frequency of patient-perceived hyperglycaemia was significantly lower in the oral semaglutide group than in the placebo group ( $p<0.0001$ for both estimands), as measured by use of the DTSQs. Outcomes for the other domains of these patientreported outcome measures were similar between treatments (appendix $\mathrm{p}$ 24).

Safety outcomes are summarised in table 2 and further information can be found in the appendix (pp 12-14). A higher proportion of participants reported an adverse event with oral semaglutide (120 [74\%] of 163) than did with placebo (105 [65\%] of 161), with a similar proportion in each group reporting serious adverse events (17 [10\%] vs 17 [11\%]). The most frequent adverse events were mild-to-moderate gastrointestinal events, primarily nausea. Nausea was more common in patients who were given oral semaglutide who had stage 3A chronic kidney disease than in those with stage 3B disease (post-hoc analysis; appendix p 25). The proportion of participants who discontinued study drug early because of adverse events was higher in the oral semaglutide group than in the placebo group (24 [15\%] vs eight [5\%]), mainly due to gastrointestinal events (principally, nausea, vomiting, abdominal pain, and dyspepsia; table 2). Three deaths occurred during the in-trial period (ie, from randomisation until death of the participant). One patient in each group died of cardiovascular causes (acute myocardial infarction in a participant in the oral semaglutide group who had a history of previous myocardial infarction, and sudden cardiac death in a participant in the placebo group who had a history of cardiovascular disease). Neither death was considered to be treatment related. Another participant in the placebo group died without a confirmed cause of death.

Overall, renal function was unchanged throughout the trial period in both treatment groups (appendix p 26); median eGFR ratios (week 31 follow-up to baseline) were 1.02 (range $0.27-1.96$ ) for oral semaglutide and 1.00 (0.68-2.17) for placebo. Geometric mean UACRs (ratio of week 26 to baseline) decreased in the oral semaglutide group (0.86 [range 0.04-56.71] but increased in the placebo group (1.19 [0.01-79.59]; appendix p 27). Two patients in the oral semaglutide group had three non-serious EAC-confirmed events of acute kidney injury (stage 1, recovered or recovering while remaining on study drug). One patient in the placebo group had a non-serious EAC-confirmed event of acute kidney injury (stage 2) and recovered.

The proportion of participants with a symptomatic hypoglycaemic episode confirmed by their blood glucose concentration while on study drug was low (nine [6\%] in the oral semaglutide group vs three [2\%] in the placebo group). No severe hypoglycaemic events occurred. EAC-confirmed cardiovascular events (five [3\%] in the oral semaglutide group vs three [2\%] in the placebo group) and diabetic retinopathy-related adverse events (five [3\%] vs two [1\%]) during the in-trial period were infrequent with both oral semaglutide and placebo. All cases of retinopathy were non-serious and mild or moderate in severity, and none required treatment or led to discontinuation of study drug. Most of these events were discovered during routine end-of-treatment eye examination and were diagnosed as non-proliferative diabetic retinopathy. No clinically relevant changes in laboratory assessments, physical examinations, or ECGs were recorded. Mean systolic blood pressure decreased by $7 \mathrm{~mm} \mathrm{Hg}$ from baseline to week 26 in the oral semaglutide group, compared with no change in the placebo group (ETD $-7 \mathrm{~mm} \mathrm{Hg}, 95 \% \mathrm{Cl}-9$ to -4 ; p<0.0001; appendix p 15). Mean diastolic blood pressure decreased by $2 \mathrm{~mm} \mathrm{Hg}$ from baseline to week 26 with oral semaglutide and increased by $1 \mathrm{~mm} \mathrm{Hg}$ with placebo (ETD $-3 \mathrm{~mm} \mathrm{Hg}, 95 \% \mathrm{Cl}-5$ to -1 ; p=0.0018; appendix p 15). Mean pulse rate was not significantly increased from baseline to week 26 with oral semaglutide (1 beat per min) compared with placebo ( 0 beats per min; ETD 1 beat per min; 95\% $\mathrm{Cl}-1$ to $2 ; \mathrm{p}=0 \cdot 56$ ).

Sensitivity analyses of the primary and confirmatory secondary endpoint supported the results of the confirmatory analysis (appendix p 17). Observed changes in $\mathrm{HbA}_{1 \mathrm{c}}$ and bodyweight over time were similar for participants in both eGFR subgroups (post-hoc analysis; appendix $\mathrm{p}$ 18). 


\section{Discussion}

In this randomised, phase $3 a$ trial, oral semaglutide was superior to placebo in decreasing $\mathrm{HbA}_{1 \mathrm{c}}$ and bodyweight at 26 weeks in patients with type 2 diabetes and moderate renal impairment who were receiving standard glucoselowering medication (treatment policy estimand). Using the trial product estimand, the ETD for $\mathrm{HbA}_{1 \mathrm{c}}$ was $-1 \cdot 0$ percentage point and for bodyweight was $-2.7 \mathrm{~kg}$ with oral semaglutide compared with placebo, and approximately $58 \%$ of participants in the oral semaglutide group met the target of $\mathrm{HbA}_{1 c}$ of less than $7.0 \%$ at 26 weeks. Additionally, in the oral semaglutide group $60 \%$ or more of participants by use of both estimands achieved a decrease in $\mathrm{HbA}_{1 \mathrm{c}}$ of 1.0 percentage point or more.

Type 2 diabetes is a common cause of chronic kidney disease, and both conditions are associated with increased cardiovascular risk, especially in combination. ${ }^{10}$ In the SUSTAIN-6 trial, ${ }^{24} 83 \%$ (2735 of 3297) of patients had cardiovascular disease, chronic kidney disease, or both, and subcutaneous semaglutide improved cardiovascular outcomes (significantly decreasing the composite incidence of cardiovascular death, non-fatal myocardial infarction, and non-fatal stroke) and led to decreased rates of nephropathy progression compared with placebo. ${ }^{24}$ Oral semaglutide is the first oral GLP-1 receptor agonist and, once available, might be preferred to injections by some patients. Like liraglutide and subcutaneous semaglutide, the pharmacokinetics of oral semaglutide are not substantially affected by renal impairment, ${ }^{11,12,25}$ making it potentially suitable for patients with chronic kidney disease.

Renal impairment restricts the choice and efficacy of medication for glycaemic control in patients with chronic kidney disease. However, unlike SGLT2 inhibitors (contraindicated when eGFR is below $45 \mathrm{~mL} / \mathrm{min}$ per $1.73 \mathrm{~m}^{2}$ ) and metformin (dose adjusted when eGFR is below $60 \mathrm{~mL} / \mathrm{min}$ per $1.73 \mathrm{~m}^{2}$ and contraindicated when it is below 30 $\mathrm{mL} / \mathrm{min}$ per $1.73 \mathrm{~m}^{2}$ ), some GLP-1 receptor agonists, including subcutaneous semaglutide, can be used without dose adjustment in patients with creatinine clearance as low as $15 \mathrm{~mL} / \mathrm{min}$ per $1.73 \mathrm{~m}^{2} .{ }^{10,11} \mathrm{In}$ addition to their potential for improved renal safety and convenience in patients with chronic kidney disease, GLP-1 receptor agonists provided better glycaemic control and weight loss than regimens containing insulin. ${ }^{26,27}$

Unlike SGLT2 inhibitors, DPP-4 inhibitors such as sitagliptin, have a wider indication for use in the setting of renal impairment. However, a meta-analysis of trials in patients with type 2 diabetes with moderate-to-severe chronic kidney disease indicated that only modest decreases in $\mathrm{HbA}_{1 \mathrm{c}}$ are achieved with DPP-4 inhibitor therapy. ${ }^{28}$ In a headto-head comparison, albiglutide was significantly better for glycaemic control and weight loss than sitagliptin in patients with chronic kidney disease, with similar tolerability. ${ }^{29}$ In the PIONEER 3 trial, ${ }^{7}$ oral semaglutide was superior to sitagliptin for glycaemic control and decreasing bodyweight, albeit in a population with normal renal function.

The results presented here for PIONEER 5 were achieved with few hypoglycaemic episodes, an important advantage over insulin and sulfonylureas, and are consistent with the results of the randomised, placebo-controlled, 26-week PIONEER 1 trial. ${ }^{6}$ In PIONEER 1, compared with placebo oral semaglutide 14 mg monotherapy resulted in decreases of $1.5 \%$ (SE 0.1) in $\mathrm{HbA}_{1 \mathrm{c}}$ and $4.1 \mathrm{~kg}$ (SE 0.3) in bodyweight, and approximately 80\% of patients achieved the target of $\mathrm{HbA}_{1 \mathrm{c}}$ of less than $7.0 \%$ (according to the trial product estimand). ${ }^{6}$ The greater magnitude of response in PIONEER 1 compared with the present study is probably because of differences in patient populations and trial design-eg, patients in PIONEER 1 were on average younger and had a shorter duration of diabetes than those in PIONEER 5, and were not receiving background glucose-lowering medications. Moreover, patients with an eGFR of less than $60 \mathrm{~mL} / \mathrm{min}$ per $1.73 \mathrm{~m}^{2}$ were excluded from PIONEER 1 and we had no such exclusion criterion herein. ${ }^{6}$

The optimal target for $\mathrm{HbA}_{1 \mathrm{c}}$ in type 2 diabetes associated with kidney disease is uncertain. Meta-analyses ${ }^{30,31}$ of studies that used intensive control (target $\mathrm{HbA}_{1 \mathrm{c}} 6 \cdot 1-7 \cdot 1 \%$ [43-54 mmol/mol]) have found that microalbuminuria and 
macroalbuminuria were decreased in patients with type 2 diabetes, but without substantial effect on downstream clinical outcomes, such as progression to end-stage kidney disease or death due to renal disease. However, another meta-analysis ${ }^{32}$ of four large randomised trials indicated clinical benefits on renal outcomes with tight glycaemic control, although glycaemic targets varied between the studies. Notably, the patients analysed in all these metaanalyses were given older classes of glucose-lowering medication, and different effects could be expected with GLP1 receptor agonists.

In the present trial, oral semaglutide had acceptable safety and tolerability in most patients, and the adverse event profile was consistent with a type 2 diabetes population with moderate renal impairment and comorbidities. Therefore, the proportions of serious adverse events were higher (in both groups) than in PIONEER $1 .{ }^{6}$ The proportions of participants who discontinued study drug early due to adverse events with oral semaglutide and placebo (15\% vs 5\%) were approximately double those in PIONEER 1 (7\% vs 2\%), ${ }^{6}$ but similar to those with liraglutide versus placebo in the LIRA-RENAL trial (14\% vs 3\%). ${ }^{26}$ Few symptomatic hypoglycaemic episodes occurred with confirmation by blood glucose concentration and none were severe. Unlike in LIRA-RENAL, ${ }^{26}$ pulse rate was not significantly increased with GLP-1 receptor agonist therapy, but significant increases in pulse rate have been reported with oral semaglutide in other trials. ${ }^{6,7}$

As in PIONEER $1^{6}$ and other phase 3 trials of oral semaglutide, ${ }^{7}$ a 4 -week dose-escalation schedule was used to minimise the anticipated gastrointestinal adverse events associated with GLP-1 receptor agonists. Similar to LIRARENAL, ${ }^{26}$ gastrointestinal events were the most common adverse events, and these mostly manifested as mild-tomoderate and transient nausea. In the present study, nausea appeared to be more common in participants with better (stage 3A vs stage 3B chronic kidney disease) renal function. Although this result might have been a chance finding due to low patient numbers, it is consistent with the observation that gastrointestinal events were more common in patients with stage 3A versus stage 3B chronic kidney disease in LIRA-RENAL. ${ }^{26}$ Few new or worsening episodes of diabetic retinopathy occurred during the trial, and no cases of proliferative retinopathy occurred.

All patients in this trial had moderate renal impairment. Exploratory observations suggested that decreases in $\mathrm{HbA}_{1 \mathrm{c}}$ and bodyweight seemed to be broadly consistent regardless of whether participants had disease at CKD-EPI stage $3 \mathrm{~A}$ or $3 \mathrm{~B}$. Overall, eGFR levels remained generally constant during the trial period and in both chronic kidney disease subgroups (stage 3A and 3B). In the LEADER cardiovascular outcomes trial, ${ }^{33}$ patients with an eGFR of 30$60 \mathrm{~mL} / \mathrm{min}$ per $1.73 \mathrm{~m}^{2}$ at baseline who were given liraglutide had significantly slower deterioration in renal function than those who were given placebo. Similarly, in the AWARD-7 study, ${ }^{27}$ dulaglutide was associated with less of a decrease in eGFR compared with insulin glargine in patients with an eGFR of $15-60 \mathrm{~mL} / \mathrm{min}$ per $1.73 \mathrm{~m}^{2}$. Although PIONEER 5 was a smaller and shorter trial than AWARD-7, and, unlike AWARD-7, did not enrol patients with stage 4 chronic kidney disease, these data collectively suggest a renoprotective effect that might apply to the GLP-1 receptor agonist class as a whole.

As observed in other trials of GLP-1 receptor agonists, including liraglutide ${ }^{26}$ and subcutaneous semaglutide, ${ }^{34}$ oral semaglutide might have a positive effect on albuminuria. UACR, a risk marker for cardiovascular disease and kidney damage, ${ }^{35,36}$ decreased during the current trial in the oral semaglutide group (based on the numerical ratio at week 26 to baseline). However, we did not do a statistical analysis of this marker and more detailed study is needed to confirm the effect of oral semaglutide in association with albuminuria and clinical outcomes in patients with chronic kidney disease.

Mean diastolic and systolic blood pressure were decreased with oral semaglutide compared with placebo, which could be beneficial in populations with longstanding diabetes and chronic kidney disease, often combined with hypertension. 
A potential limitation of the current trial is that the efficacy and safety of oral semaglutide in patients with moderate renal impairment was assessed against placebo rather than an active comparator. However, all participants were receiving standard glucose-lowering drugs as background medication. Additionally, the trial size and duration were planned to allow analysis of glucose control and not renal safety and efficacy, which require further evaluation. Although the duration of PIONEER 5 was only 26 weeks, further PIONEER trials have investigated the efficacy and safety of oral semaglutide for treatment durations of up to 78 weeks, albeit not in patients with renal impairment. ${ }^{7}$

In conclusion, once-daily oral semaglutide $14 \mathrm{mg}$ was superior to placebo in decreasing $\mathrm{HbA}_{1 \mathrm{c}}$ and bodyweight in patients with type 2 diabetes and moderate renal impairment. The overall safety profile, including renal safety, was consistent with that seen for other GLP-1 receptor agonists, and few hypoglycaemic episodes occurred. Oral semaglutide appears to provide an important addition to the currently suboptimal treatment options for patients with type 2 diabetes and moderate renal impairment. 


\section{Contributors}

OM, JWE, SH, RP, TS, and CD contributed to data collection as study investigators. TMB, SR, and OHH contributed to trial design and data analysis as employees of the funder. All authors interpreted the data and participated in writing of the manuscript, with the support of medical writing services provided by the funder. All authors read and approved the submitted version of the manuscript.

\section{Declaration of interests}

OM has been a member of the Advisory Board for Novo Nordisk, Eli Lilly, Sanofi, MSD, Boehringer Ingelheim, and AstraZeneca; has had grants paid to their institution as a study physician from AstraZeneca; received a research grant paid to their institution from Novo Nordisk; and has been a member of the Speaker's Bureau for AstraZeneca, Novo Nordisk, Eli Lilly, Sanofi, MSD, and Boehringer Ingelheim. TMB and SR are employees of Novo Nordisk A/S. JWE reports personal fees from Novo Nordisk, AstraZeneca, Bayer, MSD and Sanofi, and grants from AstraZeneca and Bristol Myers Squibb. SH reports fees paid to their institution for consultancy from Eli Lilly, Novo Nordisk, Sanofi Aventis, Zealand Pharma, Boehringer Ingelheim, UNEEG medical, and Takeda, and has been a member of the Speaker's Bureau for Eli Lilly and Novo Nordisk. OHH is an employee of and holds shares in Novo Nordisk AVS. RP reports grants and fees for lecturing and consultancy paid to their institution from AstraZeneca, Boehringer Ingelheim, Eisai, GlaxoSmithKline, Glytec, Janssen, Lexicon Pharmaceuticals, Ligand Pharmaceuticals, Eli Lilly, Merck, Mundipharma, Novo Nordisk, Pfizer, Sanofi Aventis US, Takeda, and Sanofi US Services. TS reports grants paid to their institution as a study physician from Novo Nordisk. CD reports grants, personal fees, and non-financial support from Novo Nordisk, and grants from the US National Institutes of Health, REPOWER, Sanofi, and Theracos.

\section{Data sharing}

Data will be shared with researchers submitting a research proposal approved by the independent review board. Access request proposals can be found at www.novonordisk-trials.com. Data will be made available after research completion, and approval of the product and product use in the European Union and the USA. Individual participant data will be shared in data sets in a de-identified and anonymised format, with no limitations on how the data can be used.

\section{Acknowledgments}

This trial was funded by Novo Nordisk A/S, Denmark. We thank the patients who took part in this trial, the investigators, all trial site staff, and all Novo Nordisk employees involved. We thank Emisphere (Roseland, NJ, USA) for providing a license to the Eligen Technology, the sodium N-(8-[2-hydroxybenzoyl]amino) caprylate component of oral semaglutide. We also thank Stephen Purver of Spirit Medical Communications Group for medical writing and editorial assistance (funded by Novo Nordisk A/S) and Brian Bekker Hansen of Novo Nordisk for critically reviewing the manuscript. 


\section{References}

1. DeFronzo RA. Banting lecture. From the triumvirate to the ominous octet: a new paradigm for the treatment of type 2 diabetes mellitus. Diabetes 2009; 58: 773-95.

2. Davies MJ, D'Alessio DA, Fradkin J, et al. Management of hyperglycaemia in type 2 diabetes, 2018. A consensus report by the American Diabetes Association (ADA) and the European Association for the Study of Diabetes (EASD). Diabetologia 2018; 61: 2461-98.

3. Aroda VR, Ahmann A, Cariou B, et al. Comparative efficacy, safety, and cardiovascular outcomes with onceweekly subcutaneous semaglutide in the treatment of type 2 diabetes: insights from the SUSTAIN 1-7 trials. Diabetes Metab 2019; published online Jan 4. DOI:10.1016/j.diabet.2018.12.001

4. Jensen L, Helleberg H, Roffel A, et al. Absorption, metabolism and excretion of the GLP-1 analogue semaglutide in humans and nonclinical species. Eur J Pharm Sci 2017; 104: 31-41.

5. Buckley ST, Bakdal TA, Vegge A, et al. Transcellular stomach absorption of a derivatized glucagon-like peptide-1 receptor agonist. Sci Transl Med 2018; 10: eaar7047.

6. Aroda VR, Rosenstock J, Terauchi Y, et al. Effect and safety of oral semaglutide monotherapy in type 2 diabetes_PIONEER 1 trial. Diabetes 2018; 67 (suppl 1): 2-LB (abstr).

7. Rosenstock J, Allison D, Birkenfeld AL, et al. Effect of additional oral semaglutide vs sitagliptin on glycated hemoglobin in adults with type 2 diabetes uncontrolled with metformin alone or with sulfonylurea: the PIONEER 3 randomized clinical trial. JAMA 2019; published online Mar 23. DOI:10.1001/jama.2019.2942.

8. Abdi H, Azizi F, Amouzegar A. Insulin monotherapy versus insulin combined with other glucose-lowering agents in type 2 diabetes: a narrative review. Int J Endocrinol Metab 2018; 16: e65600.

9. Edridge $\mathrm{CL}$, Dunkley AJ, Bodicoat $\mathrm{DH}$, et al. Prevalence and incidence of hypoglycaemia in 532,542 people with type 2 diabetes on oral therapies and insulin: a systematic review and meta-analysis of population based studies. PLoS One 2015; 10: e0126427.

10. Ioannidis I. Diabetes treatment in patients with renal disease: Is the landscape clear enough? World J Diabetes 2014; 5: 651-58.

11. Davies M, Chatterjee S, Khunti K. The treatment of type 2 diabetes in the presence of renal impairment: what we should know about newer therapies. Clin Pharmacol 2016; 8: 61-81.

12. Granhall C, Sondergaard FL, Thomsen M, Anderson TW. Pharmacokinetics, safety and tolerability of oral semaglutide in subjects with renal impairment. Clin Pharmacokinet 2018; 57: 1571-80.

13. International Council on Harmonisation of Technical Requirements for Registration of Pharmaceuticals for Human Use. ICH harmonised tripartite guideline. Statistical principles for clinical trials E9: Feb 5, 1998. https://www.ich.org/fileadmin/Public_Web_Site/ICH_Products/Guidelines/Efficacy/E9/Step4/E9_Guideline. pdf (accessed May 2, 2019).

14. European Medicines Agency. Ozempic summary of product characteristics. 2018. https://www.ema.europa.eu/en/documents/ product-information/ozempic-epar-product-information_en.pdf (accessed May 28, 2019).

15. International Council on Harmonisation of Technical Requirements for Registration of Pharmaceuticals for Human Use. ICH harmonised guideline E9(R1): Addendum: statistical principles for clinical trials. 2018. http://www.ich.org/products/guidelines/ efficacy/article/efficacy-guidelines.html (accessed May 2, 2019).

16. National Kidney Foundation. K/DOQI clinical practice guidelines for chronic kidney disease: evaluation, classification, and stratification. Am J Kidney Dis 2002; 39 (suppl 1): S1-266. 
17. Levey AS, Stevens LA, Schmid $\mathrm{CH}$, et al. A new equation to estimate glomerular filtration rate. Ann Intern Med 2009; 150: 604-12.

18. McMurray JJ, Adamopoulos S, Anker SD, et al. ESC guidelines for the diagnosis and treatment of acute and chronic heart failure 2012: The Task Force for the Diagnosis and Treatment of Acute and Chronic Heart Failure 2012 of the European Society of Cardiology. Developed in collaboration with the Heart Failure Association (HFA) of the ESC. Eur J Heart Fail 2012; 14: 803-69.

19. Davies M, Pieber TR, Hartoft-Nielsen ML, Hansen OKH, Jabbour S, Rosenstock J. Effect of oral semaglutide compared with placebo and subcutaneous semaglutide on glycemic control in patients with type 2 diabetes: a randomized clinical trial. JAMA 2017; 318: 1460-70.

20. Inzucchi SE, Bergenstal RM, Buse JB, et al. Management of hyperglycaemia in type 2 diabetes, 2015: a patient-centred approach. Update to a position statement of the American Diabetes Association and the European Association for the Study of Diabetes. Diabetologia 2015; 58: 429-42.

21. American Diabetes Association. 8. Pharmacologic approaches to glycemic treatment: standards of medical care in diabetes-2018. Diabetes Care 2018; 41 (suppl 1): S73-85.

22. Garber AJ, Abrahamson MJ, Barzilay JI, et al. Consensus statement by the American Association of Clinical Endocrinologists and American College of Endocrinology on the comprehensive type 2 diabetes management algorithm - 2018 executive summary. Endocr Pract 2018; 24: 91-120.

23. Seaquist ER, Anderson J, Childs B, et al. Hypoglycemia and diabetes: a report of a workgroup of the American Diabetes Association and the Endocrine Society. Diabetes Care 2013; 36: 1384-95.

24. Marso SP, Bain SC, Consoli A, et al. Semaglutide and cardiovascular outcomes in patients with type 2 diabetes. N Engl J Med 2016; 375: 1834-44.

25. Marbury TC, Flint A, Jacobsen JB, Derving Karsbol J, Lasseter K. Pharmacokinetics and tolerability of a single dose of semaglutide, a human glucagon-like peptide-1 analog, in subjects with and without renal impairment. Clin Pharmacokinet 2017; 56: 1381-90.

26. Davies MJ, Bain SC, Atkin SL, et al. Efficacy and safety of liraglutide versus placebo as add-on to glucoselowering therapy in patients with type 2 diabetes and moderate renal impairment (LIRA-RENAL): a randomized clinical trial. Diabetes Care 2016; 39: 222-30.

27. Tuttle KR, Lakshmanan MC, Rayner B, et al. Dulaglutide versus insulin glargine in patients with type 2 diabetes and moderate-to-severe chronic kidney disease (AWARD-7): a multicentre, open-label, randomised trial. Lancet Diabetes Endocrinol 2018; 6: 605-17.

28. Singh-Franco D, Harrington C, Tellez-Corrales E. An updated systematic review and meta-analysis on the efficacy and tolerability of dipeptidyl peptidase-4 inhibitors in patients with type 2 diabetes with moderate to severe chronic kidney disease. SAGE Open Med 2016; 4: 2050312116659090.

29. Leiter LA, Carr MC, Stewart M, et al. Efficacy and safety of the once-weekly GLP-1 receptor agonist albiglutide versus sitagliptin in patients with type 2 diabetes and renal impairment: a randomized phase III study. Diabetes Care 2014; 37: 2723-30.

30. Coca SG, Ismail-Beigi F, Haq N, Krumholz HM, Parikh CR. Role of intensive glucose control in development of renal end points in type 2 diabetes mellitus: systematic review and meta-analysis intensive glucose control in type 2 diabetes. Arch Intern Med 2012; 172: 761-69.

31. Rodriguez-Gutierrez R, Montori VM. Glycemic control for patients with type 2 diabetes mellitus: our evolving faith in the face of evidence. Circ Cardiovasc Qual Outcomes 2016; 9: 504-12. 
32. Zoungas S, Arima H, Gerstein HC, et al. Effects of intensive glucose control on microvascular outcomes in patients with type 2 diabetes: a meta-analysis of individual participant data from randomised controlled trials. Lancet Diabetes Endocrinol 2017; 5: 431-37.

33. Mann JFE, Orsted DD, Brown-Frandsen K, et al. Liraglutide and renal outcomes in type 2 diabetes. $N$ Engl J Med 2017; 377: 839-48.

34. Bloomgarden Z. The kidney and cardiovascular outcome trials. J Diabetes 2018; 10: 88-89.

35. Gerstein HC, Mann JF, Yi Q, et al. Albuminuria and risk of cardiovascular events, death, and heart failure in diabetic and nondiabetic individuals. JAMA 2001; 286: 421-26.

36. Schmieder RE, Mann JF, Schumacher H, et al. Changes in albuminuria predict mortality and morbidity in patients with vascular disease. J Am Soc Nephrol 2011; 22: 1353-64. 
Figure 1: Trial profile

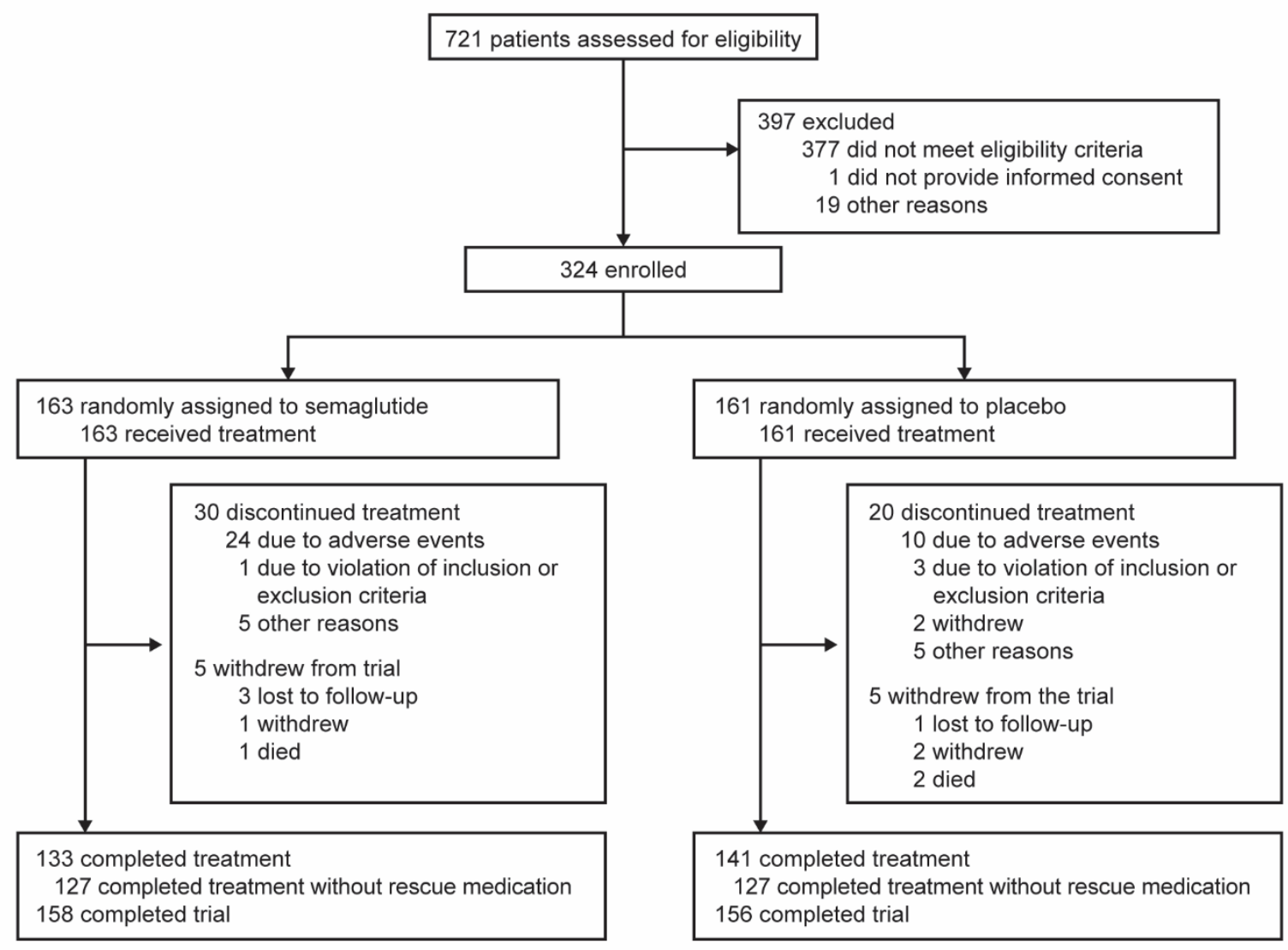


Figure 2: Mean change in $\mathrm{HbA}_{1 c}$ up to week 26 for the treatment policy estimand $(A)$ and trial product estimand (B)

A Mean baseline $\mathrm{HbA}_{1 \mathrm{c}}: 8 \cdot 0 \%(\mathrm{SD} 0 \cdot 7 ; 64 \mathrm{mmol} / \mathrm{mol}[\mathrm{SD} 8])$
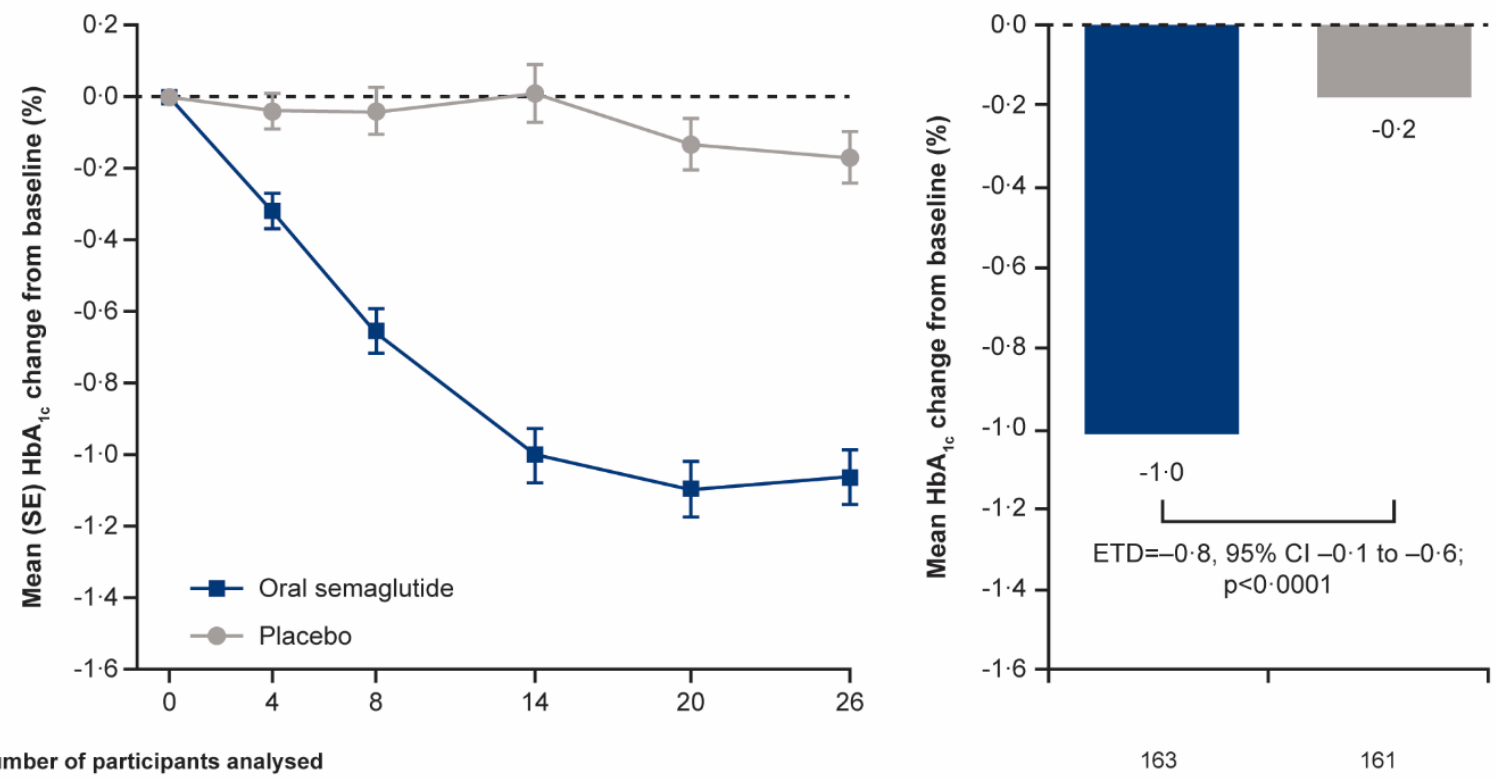

Number of participants analysed

$\begin{array}{rrrrrr}\text { Oral semaglutide } 163 & 160 & 159 & 145 & 151 & 154 \\ \text { Placebo } 161 & 155 & 153 & 146 & 148 & 155\end{array}$

B Mean baseline $\mathrm{HbA}_{1 \mathrm{C}}: 8 \cdot 0 \%(\mathrm{SD} 0 \cdot 7 ; 64 \mathrm{mmol} / \mathrm{mol}[\mathrm{SD} 8])$
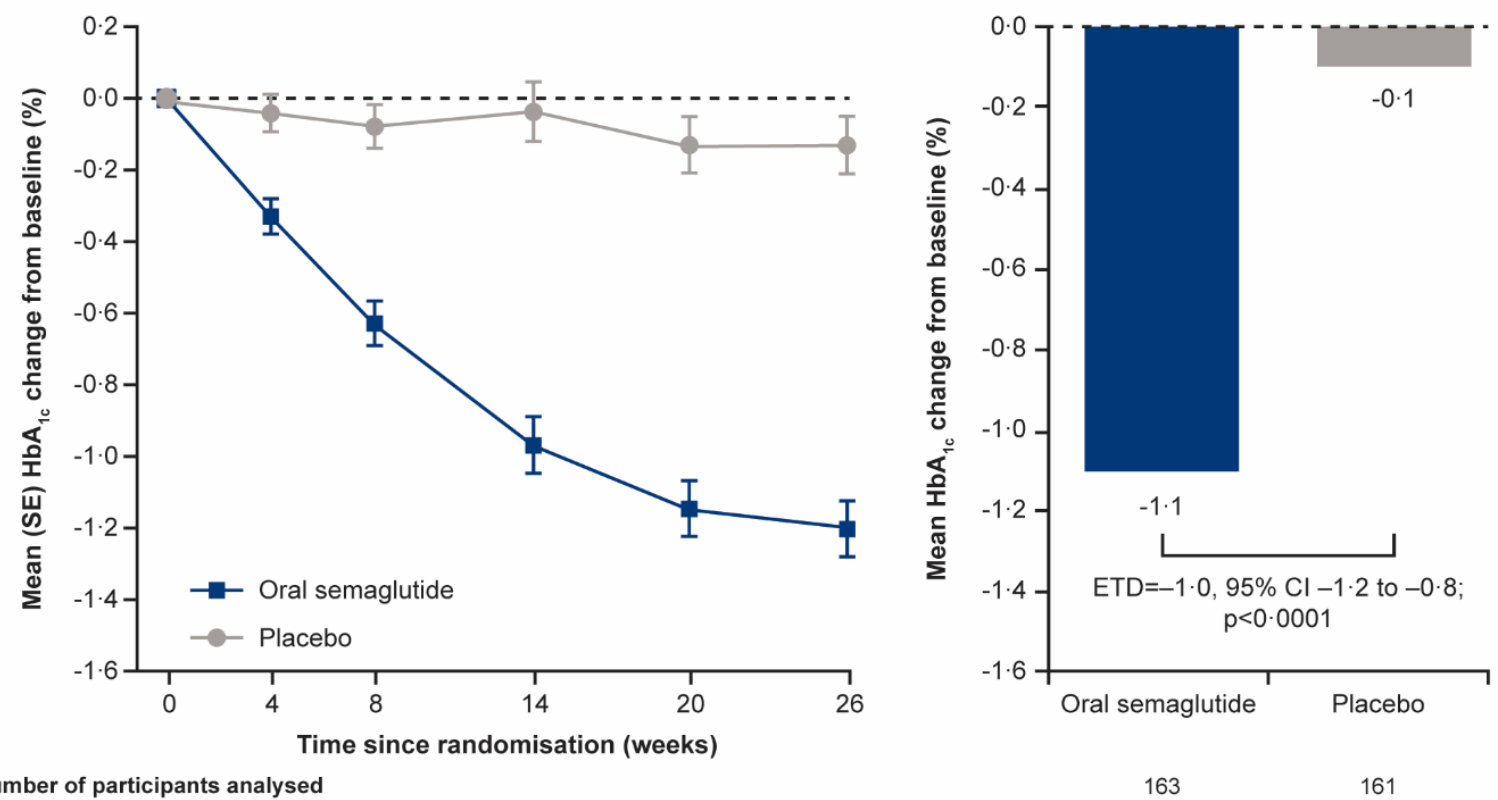

Number of participants analysed

$\begin{array}{rrrrrr}\text { Oral semaglutide 163 } & 157 & 151 & 132 & 129 & 126 \\ \text { Placebo 161 } & 152 & 148 & 136 & 130 & 127\end{array}$

Left-hand graphs are observed mean values, with error bars showing SEs. Right-hand graphs are estimated means and statistical analysis at week 26 from the primary analysis. Number of participants given is the number contributing to analysis. ETD=estimated treatment difference. $\mathrm{HbA}_{1 \mathrm{c}}=$ glycated haemoglobin. 
Figure 3: Mean change in bodyweight up to week 26 by the treatment policy estimand $(A)$ and trial product estimand $(B)$

A Mean baseline bodyweight $90.8 \mathrm{~kg}$ (SD 17.6)
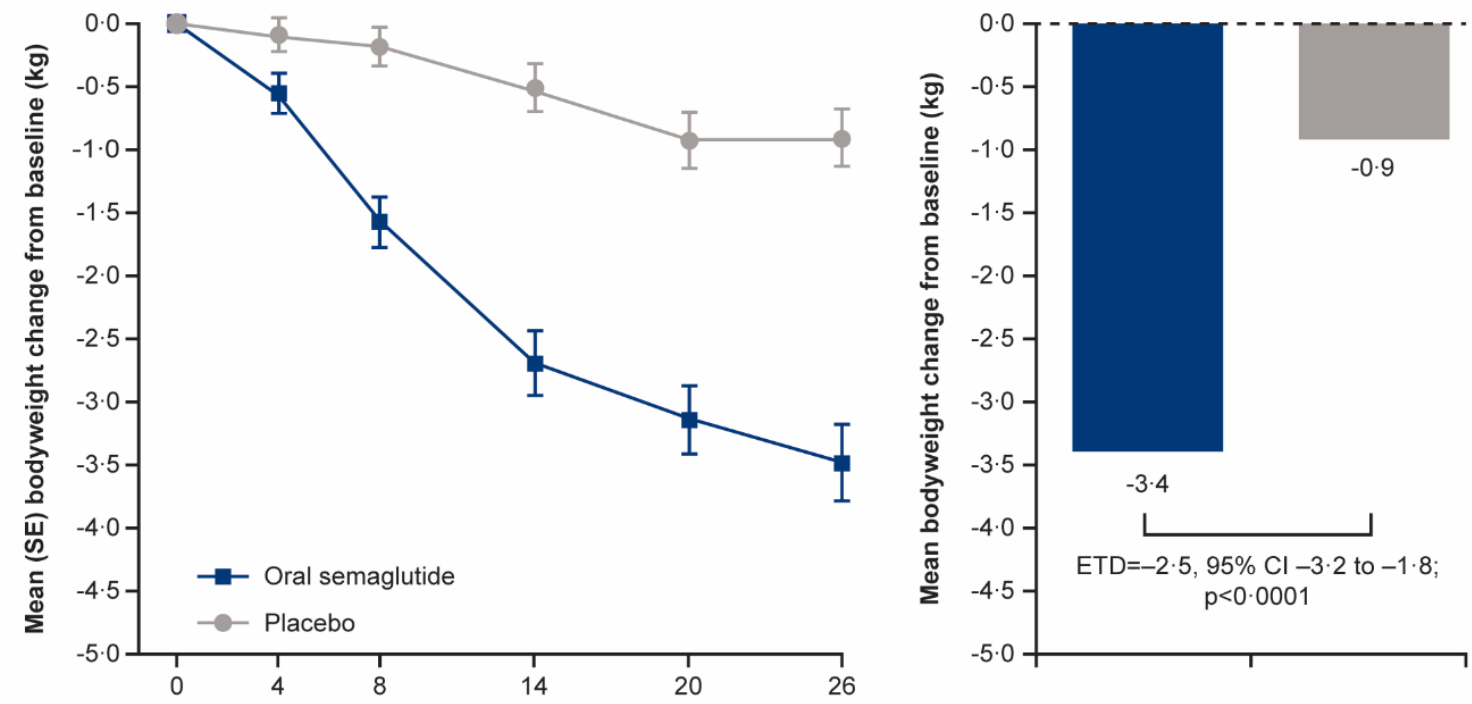

Number of participants analysed

$\begin{array}{rrrrrr}\text { Oral semaglutide } 162 & 161 & 158 & 148 & 152 & 155 \\ \text { Placebo 161 } & 157 & 156 & 150 & 148 & 155\end{array}$

162

161

B Mean baseline bodyweight $90 \cdot 8 \mathrm{~kg}(\mathrm{SD} 17 \cdot 6)$
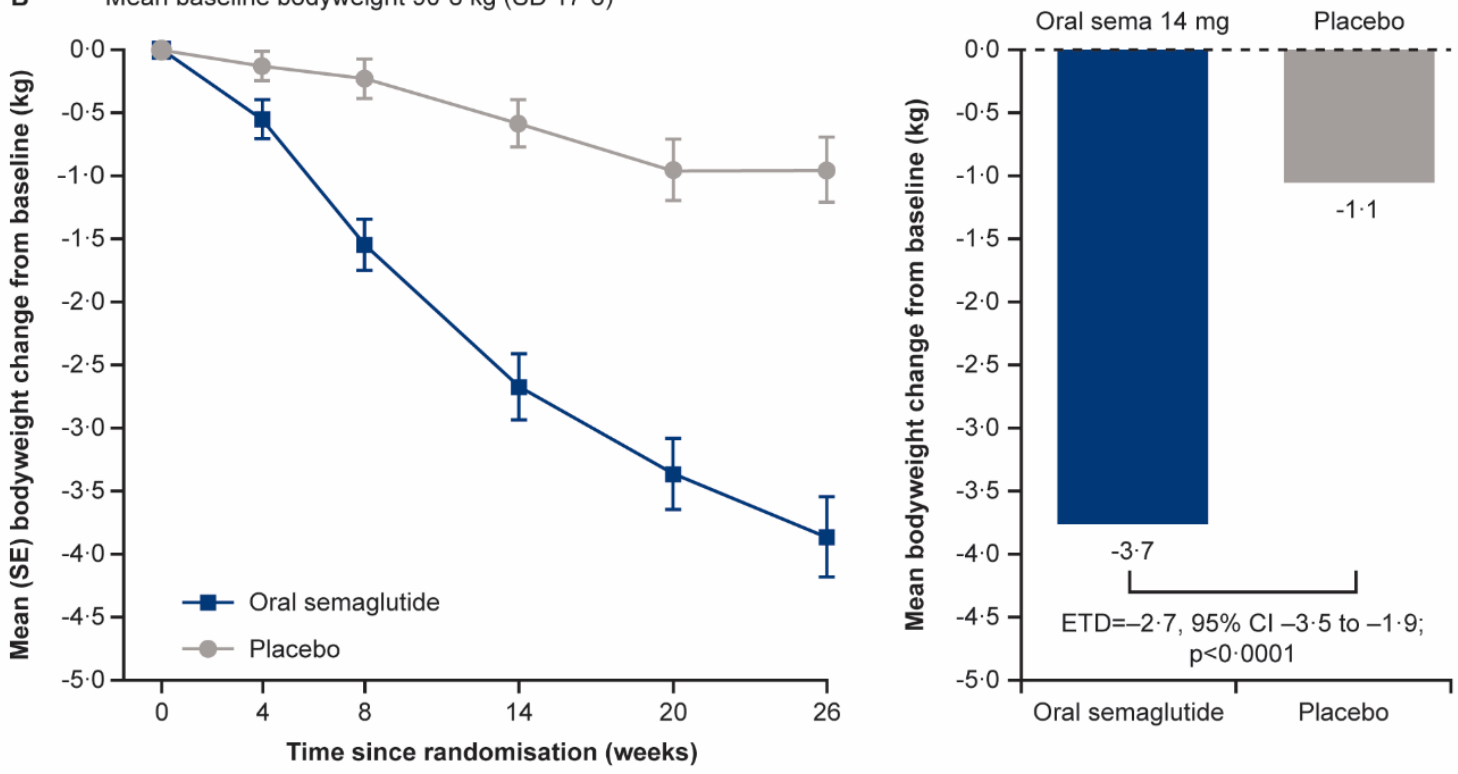

Number of participants analysed

162

161

\begin{tabular}{|c|c|c|c|c|c|}
\hline Oral semaglutide 162 & 158 & 150 & 135 & 130 & 127 \\
\hline Placebo 161 & 154 & 150 & 140 & 131 & 127 \\
\hline
\end{tabular}

Left-hand graphs are observed mean values with error bars showing SEs. Right-hand graphs are estimated means and statistical analysis at week 26 from the confirmatory secondary analysis. Numbers of participants are the number contributing to the analysis. $E T D=e s t i m a t e d$ treatment difference. 
Figure 4: Composite outcome of $\mathrm{HbA}_{1 c}$ of less than $7.0 \%$ without hypoglycaemic episodes or weight gain at week 26 by the treatment policy estimand $(A)$ and trial product estimand $(B)$

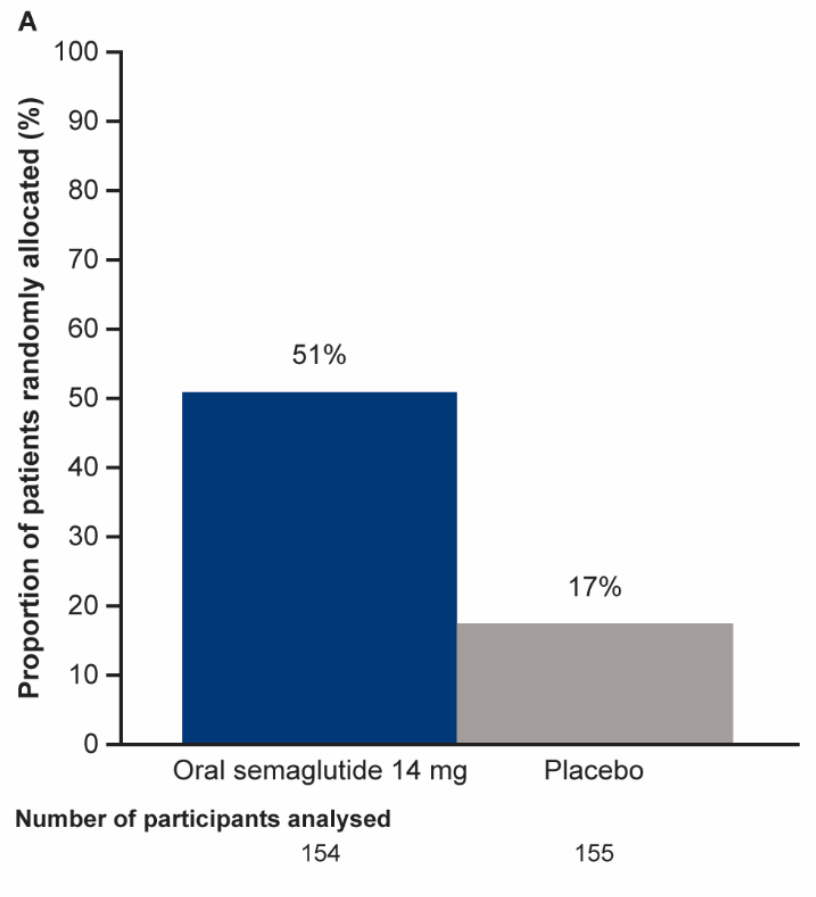

B

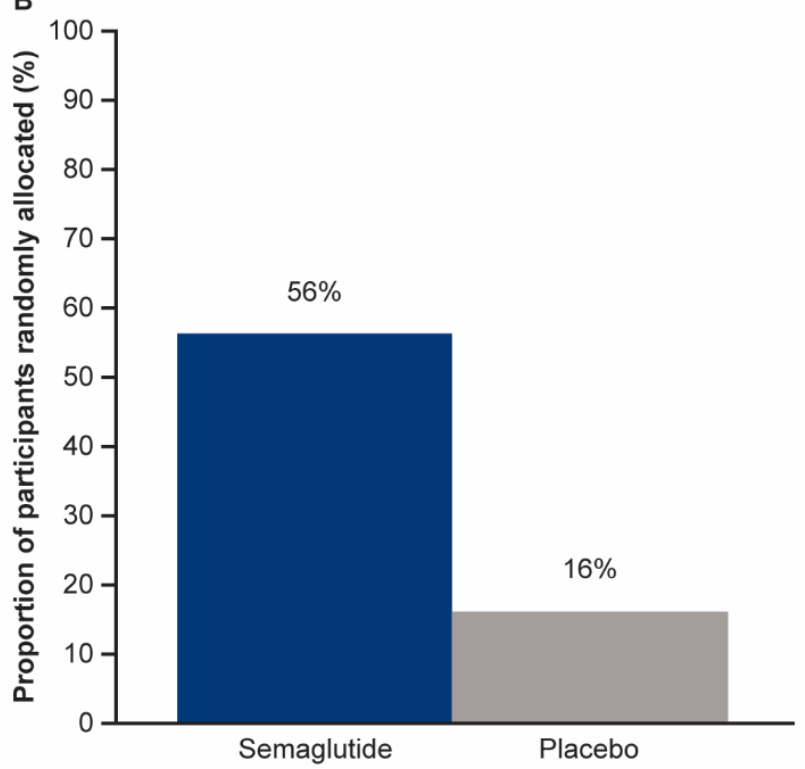

Number of participants analysed

126

127

Symptomatic hypoglycaemic events were defined as severe or confirmed by blood glucose concentration ( $3 \cdot 1 \mathrm{mmol} / \mathrm{L}[<56 \mathrm{mg} / \mathrm{dL}])$. Proportions of patients achieving the composite outcomes are based on observed data, with numbers below bars being the number of participants with non-missing information. $\mathrm{HbA}_{1 \mathrm{c}}=$ glycated haemoglobin . 
Table 1: Demographics and baseline characteristics

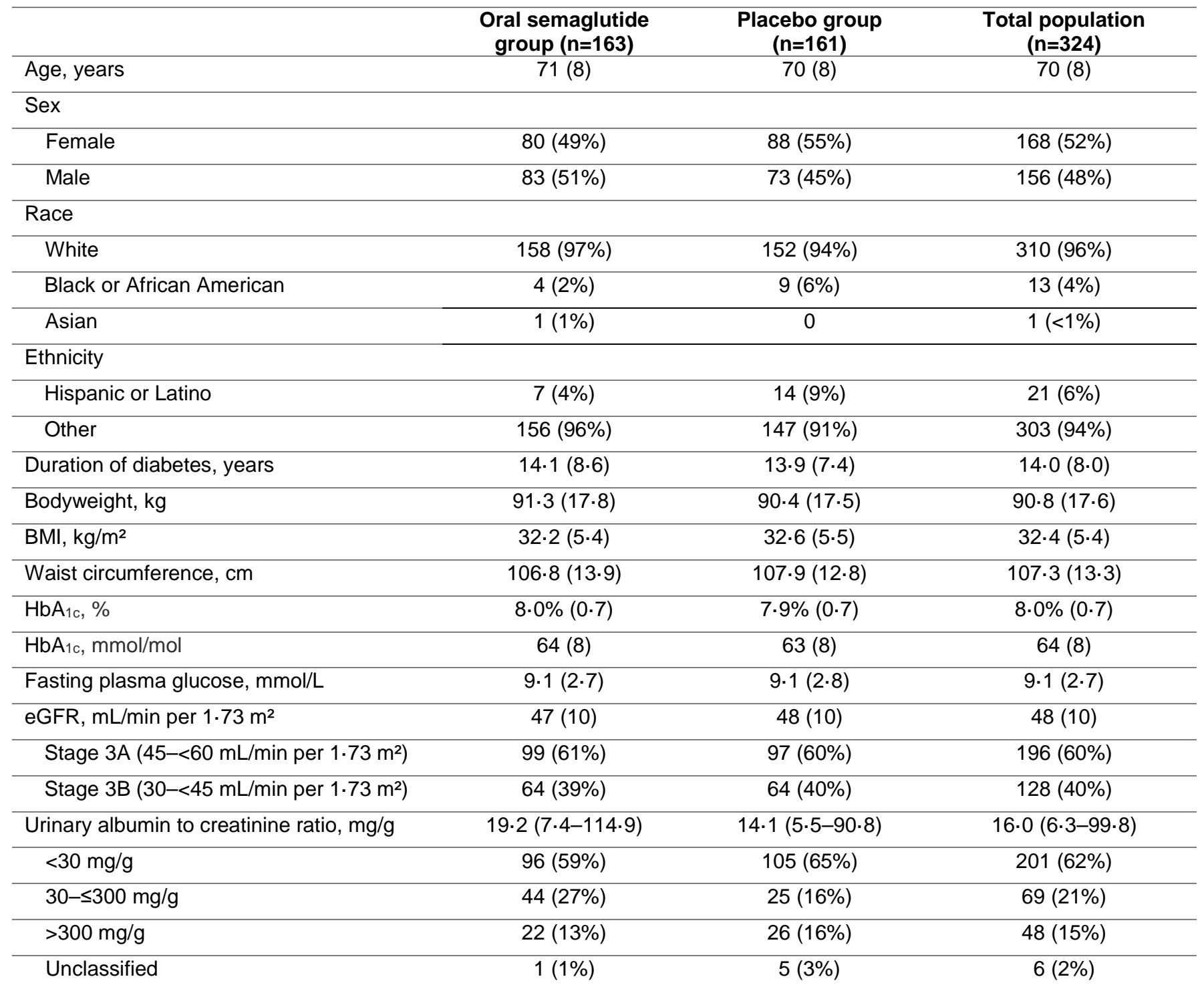

Data are mean (SD), n (\%), or median (IQR). eGFR=estimated glomerular filtration rate. $\mathrm{HbA}_{1 \mathrm{c}}=$ glycated haemoglobin. 
Table 2: Summary of adverse events

\begin{tabular}{|c|c|c|}
\hline & $\begin{array}{l}\text { Oral semaglutide } \\
\text { group }(n=163)\end{array}$ & $\begin{array}{l}\text { Placebo group } \\
(n=161)\end{array}$ \\
\hline Adverse events (in trial) & $122(75 \%)$ & $109(68 \%)$ \\
\hline Adverse events (on assigned treatment) & $120(74 \%)$ & $105(65 \%)$ \\
\hline Severe & $10(6 \%)$ & $15(9 \%)$ \\
\hline Moderate & $61(37 \%)$ & $42(26 \%)$ \\
\hline Mild & $106(65 \%)$ & $89(55 \%)$ \\
\hline \multicolumn{3}{|l|}{ Serious adverse events } \\
\hline In trial & $20(12 \%)$ & $18(11 \%)$ \\
\hline On treatment & $17(10 \%)$ & $17(11 \%)$ \\
\hline $\begin{array}{l}\text { Severe }^{\star} \text { or blood glucose-confirmed } \\
\text { hypogymptomatic } \\
\text { hypoglycaemic episode } \\
\text { (on treatment) }\end{array}$ & $9(6 \%)$ & $3(2 \%)$ \\
\hline \multicolumn{3}{|l|}{ Most frequent on-treatment adverse events ${ }^{\S}$} \\
\hline Nausea & $31(19 \%)$ & $12(7 \%)$ \\
\hline Constipation & $19(12 \%)$ & $6(4 \%)$ \\
\hline Vomiting & $19(12 \%)$ & $2(1 \%)$ \\
\hline Diarrhoea & $17(10 \%)$ & $6(4 \%)$ \\
\hline Dyspepsia & $16(10 \%)$ & $2(1 \%)$ \\
\hline Decreased appetite & $11(7 \%)$ & 0 \\
\hline Headache & $10(6 \%)$ & $8(5 \%)$ \\
\hline Back pain & $1(1 \%)$ & $9(6 \%)$ \\
\hline Discontinued study drug because of adverse events & $24(15 \%)$ & $8(5 \%)$ \\
\hline \multicolumn{3}{|c|}{ On-treatment adverse events leading to premature discontinuation of study drug } \\
\hline Gastrointestinal disorders & $19(12 \%)$ & $3(2 \%)$ \\
\hline Fatal adverse events (in trial) & $1(1 \%)$ & $2(1 \%)$ \\
\hline
\end{tabular}

Data are $\mathrm{n}(\%) .{ }^{*}$ American Diabetes Association classification. ${ }^{\dagger}$ Plasma glucose $3.1 \mathrm{mmol} / \mathrm{L}$ (<56 mg/dL); ${ }^{\ddagger}$ Hypoglycaemic episodes were reported on a separate form to adverse events. §Occurring in $>5 \%$ of patients in either group (preferred term by MedDRA

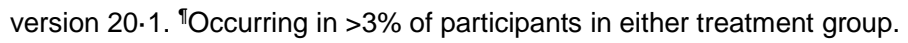

